\title{
OPTIMIZATION ASSIGNMENT OF WESTERN PATROL UNIT (KRI) ELEMENT IN SEA SECURITY OPERATIONS IN RIAU ISLANDS WATER AREAS USING SET COVERING MODEL
}

\author{
Agus Setiadji ${ }^{1}$, Didit Herdiawan ${ }^{2}$, Benny Sukandari ${ }^{3}$, Muksin ${ }^{4}$ \\ 1,2,4 Indonesia Naval Technology College, STTAL Surabaya Indonesia \\ ${ }^{3}$ Indonesia Naval Command and Staff College, Seskoal Jakarta Indonesia
}

\begin{abstract}
The territorial waters of Riau Islands are one of the locations with a high level of vulnerability to violations in the sea in Indonesia, because this region is directly adjacent to neighboring countries and is an international trade and shipping route. One of the roles of the Indonesian Navy is to maintain the security of national jurisdictions, including in the territorial waters of Riau Islands, which are then realized in Operation Sea Security, where the implementation still has several constraints, namely budget constraints, technical capabilities, number of ships, limited information, and limitations supporting facilities, so that the implementation of Marine Security Operations is not optimal. The main reason is the placement of patrol boats during operations is not well organized. With these problems, a study was conducted using the set covering method to get the most optimal location for patrol boat placement with as few ships as possible but still be able to reach the entire waters of the Riau Islands and minimize operational costs. In this study a discrete approach was taken, namely the determination of the critical points, which numbered 37 vulnerable points. All these points must be affordable by the ship on duty.
\end{abstract}

Keywords: Set Covering, Location Selection, Marine Security Operations, Riau Islands Waters Region

\section{INTRODUCTION}

The Indonesian Navy as the enforcer of state sovereignty at sea has a very important role in relation to the security of the territorial sea area of the Unitary State of the Republic of Indonesia. The security activities of this region are realized through the implementation of Marine Security Operations which are part of the Sea Combat Operation which aims to prevent and take action on any form of security disturbance in the Sea as a form of law enforcement and sovereignty in the Sea. The territorial waters of Riau Islands are one of the locations with a high level of vulnerability to violations in the sea in Indonesia, because this region is directly adjacent to neighboring countries and is an international trade and shipping route. One of the roles of the Indonesian Navy is to maintain the security of national jurisdictions, including in the territorial waters of Riau Islands, which are then realized in Operation Sea Security, where the implementation still has several constraints, namely budget constraints, technical capabilities, number of ships, limited information, and limitations supporting facilities, so that the implementation of Marine Security Operations is not optimal. The main reason is the placement of patrol boats during operations is not well organized.

With these problems, a study was conducted using the set covering method to get the most optimal location for patrol boat placement with as few ships as possible but still be able to reach the entire waters of the Riau Islands and minimize operational costs. In this study a discrete approach was taken, namely the determination of the critical points, which numbered 37 vulnerable points. All these points must be affordable by the ship on duty Based on the background of the research presented above, the problem raised in this thesis research is "How to optimize the placement of KRI element assignments in the Armabar Patrol Ship Unit in the implementation of Marine Security Operations in the territorial waters of Riau Islands using a set covering model?". 
The objectives to be achieved in this thesis research are as follows: Optimizing the use / operation of the number of Patrol Vessels to cover the entire operating sector and knowing the estimated operational costs and determine the location / sector of the patrol for Patrol Ships in optimal Marine Security Operations. The benefits that can be obtained from this Final Project research are: Providing input to decision makers, TNI leaders, especially the Western Military Cooperative Staff and Marine Security Task Force (Guskamlabar) as well as KRI (Patrol Ship) elemental commanders in determining the strategy for carrying out maritime security operations, increase success in sea security operations carried out by elements of KRI (Patrol Ships) located in the waters of the Riau Islands, save existing resources and Produce an optimization formulation that can be applied in the Coalition and Guskamlab Operations Staff in order to assign Navy patrol ships to the operating sector.

The limits used in this Final Project research are: The sea security operation was only carried out by the KRI from the Armabar patrol unit, The area that became the object of research was the waters of the Riau Islands alone,assignments carried out based on existing limitations or constraints and do not make changes to these constraints and not discussing the weaponry of the Navy Navy Patrol Ship. The assumptions used in this Final Project research are ; the boundaries of the Operational Security Sea area have not changed from before,base and Intelligence titles are ready to support operations, All elements of the Armabar Patrol ship are assumed to be ready for operation, Sea Security Operations are carried out with the condition of the State in a state of peace and Weather conditions in the Sea are calm, sea state $1-2$

\section{MATERIAL AND METHODS}

2.1 Material

2.1.1 Operation Sea Security is the operation of daily presence in the sea carried out by ships and aircraft of the Indonesian Navy's maritime patrol which have strategic value for the existence of national sovereignty and stability of security at sea in Indonesia's national jurisdiction.

2.1.2 The Patrol Ship Unit is the implementing unit tasked with developing Personnel and Material under the Fleet Command. In addition, Satrol is a unit that supports the main task of the Fleet Command, namely, among others, its involvement in the title of security operations in the territorial waters of National Jurisdiction.

2.1.3 KRI is a ship owned by the Indonesian Navy that has special signs, under the command of a Navy Officer who is manned by ABK who are subject to army discipline laws that have integrated technical requirements and requirements.

2.1.4 The covering set is part of an integer linear programming that aims to minimize the number of location points of service facilities but can serve all points of demand (Toregas, 1971). To be able to describe the set covering model can be formulated or formulated as follows:

$X=$ Facilities (ships) used by ships used will be symbolized by alphabet $A, B$, and so on until all types of vessels are represented. So $x=\{A$, $\mathrm{B}, \ldots\}$

$I=$ Critical points or alternative points of placement of ships

$j=$ Prone point

$n=$ The number of alternative points or vulnerable points that exist

$\mathrm{C}_{\mathrm{x}}=$ Ship operating costs $\mathrm{x}$.

$K_{i x}=\left\{i / d_{i j} \leq D_{x}\right\}$ that is, all points that can be reached by ship $x$ which are placed at point $i$. Where the distance between the vulnerable points occupied by the ship and the vulnerable 
points to be reached (smaller) is smaller than the distance of the ship's reach $x$.

$m_{x}=$ The number of vessels available for this type of ship $\mathrm{x}$

Decision variable:

$x_{i}\left\{\begin{array}{l}1 \\ 0\end{array}\right.$

$A_{i}$ worth 1 if at location $i$ is placed by ship $A$. $B i$ is worth 1 if at location $i$ is placed ship $B$, and so on. Value 0 if not occupied by the ship. From the above notation, the set covering model can be formulated as follows:

Minimize

$$
\sum_{\mathrm{i}=1}^{n} A_{\mathrm{i}}+\sum_{\mathrm{i}=1}^{n} B_{\mathrm{i}}+\cdots
$$

$$
\sum_{j \in R i x} A_{j}+B_{j}+\cdots \geq 1 \forall i \in n
$$

$$
A i_{v} B i_{s} \ldots\{0.1\} \forall i \in I
$$

$$
\sum_{i=1}^{n} x_{i} \leq m_{x}
$$

$$
\sum_{\mathrm{i}=1}^{n} A_{\mathrm{i}}+B_{\mathrm{i}}+\cdots \leq 1
$$

From the above formulation it can be seen that the aim (2.7) is to minimize the use of the number of vessels / facility placement. Limiter (2.8) shows that each vulnerable point can be reached by at least 1 ship. The delimiter (2.9) indicates that the decision variable is a member of a binary number. Limiting (2.10) indicates that each type of vessel must not exceed the number of vessels available. Limiter (2.11) shows that each critical point is not occupied by more than one swamp point.

\subsubsection{Distance Between Two Points}

The formula for calculating the distance between two points $P_{1}\left(x_{1}, y_{1}\right)$ and $P_{2}\left(x_{2}, y_{2}\right)$ based on Pythagoras's Theorema is

$$
\overline{P_{1} P_{2}}=\sqrt{\left(x_{2}-x_{1}\right)^{2}+\left(y_{1}-y_{1}\right)^{2}}
$$

The distance between two points is to draw the shortest line between the two points

\subsubsection{Determination of Selective Prone Areas}

Selective vulnerable areas are areas that are considered prone to the emergence of various forms of violations of sovereignty and security disturbances at sea that can harm national interests in and or by sea. The presence of Navy patrol aircraft and aircraft that are faced with limited operational facilities and infrastructure, requires an operational pattern and a strategic plan and title in the priority of marine security operations in certain waters which are considered prone to various forms of violations of sovereignty and security disturbances in the sea.

\subsubsection{Logistics Planning and Costs}

Hadi Firmanto (2006), Logistics planning is a process of strategy in regulating a procurement, transfer and storage, final inventory through company organizations and channels / civil and military agencies, so that current and future benefits can be maximized through cost effectiveness.

The logistical costs of ships and the Indonesian Navy's maritime patrol aircraft are broadly divided into two, namely liquid logistics costs and personnel logistics. Included in the liquid logistics costs are the costs of fuel, engine oil, lubricating oil, hydraulic oil and fresh water, while those that include personnel logistics costs are the cost of food during operations, screen allowances, non-screen allowances, leadership benefits, flight allowances, pocket money as well as operating vessel maintenance costs 


\subsection{Methods}

The research method is the steps taken in solving a problem faced. The type of data collected is in the form of qualitative data and quantitative data consisting of primary data and secondary data. The research method used in this thesis research is as follows:

a. This type of research used in the research on the optimization of placement of patrol boat assignments is a type of quantitative research that is research that aims to explain the differences, comparisons, relationships, or influence between two or more variables.

b. The data used in this study are secondary data in the form of data from inspection results by Patrol Ships and data from Armabar operations staff about the division of the operating sector. The data needed is the coverage coverage of the Indonesian Navy ships, vulnerable points at sea, and KRI logistics costs. So to get this data it is necessary to collect documents that record the data.

c. Data collection in this study using the documentary method is to collect data by collecting and analyzing

\subsection{Data Analysis and Interpretation} Methodse Analisis dan Interpretasi Data

After the required data is available, then the data analysis and interpretation are then carried out. In this stage the existing data will be processed in several stages as follows:

a. Calculation of Coverage of KRI Coverage, Circle KRI coverage coverage obtained through calculation. The circle radius of the KRI coverage range is assumed to be the fulfillment distance Coverage $\mathrm{KRI}\left(D_{c}\right)$.

b. Determination of Prone Location Points The point of prone location can be seen from historical data of violations. The vulnerable location points will also be an alternative location for the Marine Security Operation patrol.

c. Calculation of Distance Between Prone Locations Using the Pythagorean theorem, the distance between two points, namely between the vulnerable locations can be known. In the set covering model, this distance becomes the distance between the vulnerable points of $\mathrm{k}$ and alternative patrol locations $j\left(d_{k j}\right)$.

\section{Set Covering Model Compilation}

1. Objective function Minimize :

$z=C_{i} \sum_{i=1}^{n} A_{i}+C_{z} \sum_{i=1}^{n} B_{i}+C_{C} \sum_{i=1}^{\pi} C_{i}+C_{D} \sum_{i=1}^{n} D_{i}+C_{z} \sum_{i=1}^{n} E_{i}+C_{z} \sum_{i=1}^{n} F_{i}+C_{C} \sum_{i=1}^{n} G_{i}$

2. Decicions variable

$A i_{v} B i_{s}, C i_{s} D i_{s} E i_{s} F i_{s} G i\left\{\begin{array}{l}1 \\ 0\end{array}\right.$

3. Constrain function

a. $\sum_{j \in K i x} A_{j}+B_{j}+\cdots \geq 1 \forall i \in n$

b. $A i_{s} B i_{s} \mathrm{Ci}_{s} \mathrm{Di}, \mathrm{Ei}, \mathrm{Fi}, \mathrm{Gi}\{0,1\} \forall i$

c. $\quad \sum_{i=1}^{\mathrm{n}} x_{i} m_{x}$

d. $\quad \sum_{i=1}^{n} A_{i}+B_{i}+\cdots \leq 1$

\section{RESULT}

\subsection{Ship Ability Data}

Patrol ships assigned to the western Indonesian territorial waters consist of 7 types of vessels, totaling 20 ships. This data is obtained from the Coalarm Patrol Ship Unit. Existing ship data will be used in determining the operating costs of each ship as well as input in the preparation of the optimization model, namely the anchorage (radar). 


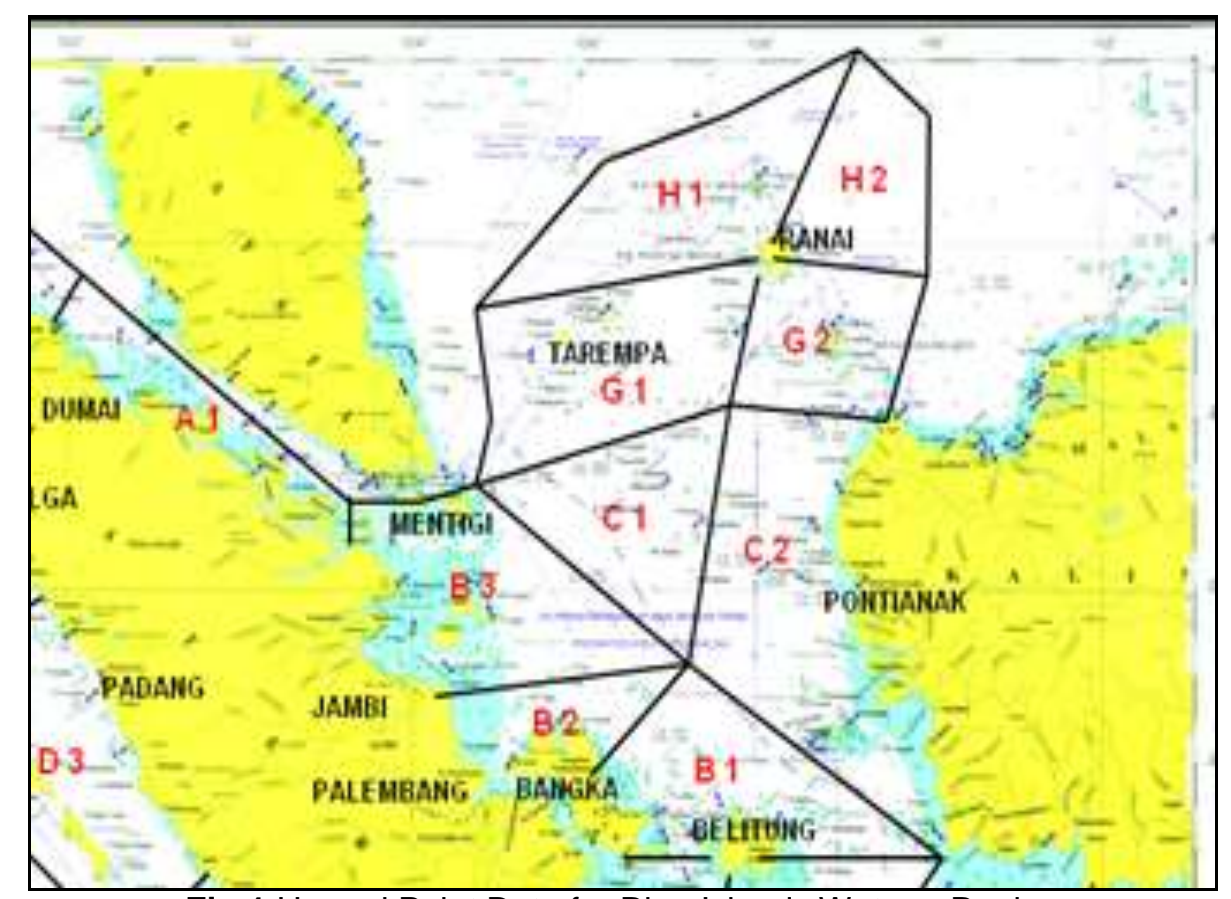

Fig.1 Hazard Point Data for Riau Islands Waters Region

\subsection{Ship operating cost}

The amount of operating costs for each ship the cost needed per unit which will be used in the calculation of each ship

is determined by the size of the unit cost, which is

Table 1. Optimization of Assignment of Indonesian Navy ships in Marine Security Operations for the Riau Islands Waters Region

\begin{tabular}{|c|c|c|c|}
\hline Ship's name & $\begin{array}{c}\text { Endurance } \\
\text { (Day) }\end{array}$ & $\begin{array}{c}\text { Person } \\
\text { number }\end{array}$ & Cost (Rp) \\
\hline A1 & 9 & 26 & 883.791 .000 \\
\hline B1 & 5 & 38 & 754.181 .500 \\
\hline B2 & 5 & 38 & 754.181 .500 \\
\hline C1 & 3 & 30 & 312.124 .800 \\
\hline C2 & 3 & 30 & 312.124 .800 \\
\hline C3 & 3 & 30 & 312.124 .800 \\
\hline C4 & 3 & 30 & 312.124 .800 \\
\hline C5 & 3 & 30 & 312.124 .800 \\
\hline C6 & 3 & 30 & 312.124 .800 \\
\hline C7 & 3 & 30 & 312.124 .800 \\
\hline C8 & 3 & 30 & 312.124 .800 \\
\hline D1 & 3 & 27 & 388.746 .000 \\
\hline D2 & 3 & 27 & 388.746 .000 \\
\hline D3 & 3 & 27 & 388.746 .000 \\
\hline E1 & 3 & 20 & 330.497 .400 \\
\hline E2 & 3 & 20 & 330.497 .400 \\
\hline E3 & 3 & 20 & 330.497 .400 \\
\hline E4 & 3 & 20 & 330.497 .400 \\
\hline F & 3 & 23 & 148.449 .000 \\
\hline G & 3 & 20 & 314.312 .400 \\
\hline
\end{tabular}

\subsection{Decicions Variable}

The decision variable is whether to place one ship at one vulnerable point or not to place the ship.
The total number of existing vessels is 20 and 37 vulnerable points. 
Table 2. Sectors of Assignment of Indonesian Navy ships in Marine Security Operations for the Riau Islands Waters Region

\begin{tabular}{|c|c|c|c|c|c|c|c|}
\hline \multirow[t]{2}{*}{ Point } & \multicolumn{7}{|c|}{ KRI Group } \\
\hline & A & B & C & D & $E$ & $F$ & G \\
\hline 1 & A1 & B1 & C1 & D1 & E1 & F1 & G1 \\
\hline 2 & A2 & B2 & C2 & D2 & E2 & F2 & G2 \\
\hline 3 & A3 & B3 & C3 & D3 & E3 & F3 & G3 \\
\hline 4 & A4 & B4 & $\mathrm{C} 4$ & D4 & $\mathrm{E} 4$ & $\mathrm{~F} 4$ & G4 \\
\hline 5 & A5 & B5 & C5 & D5 & E5 & F5 & G5 \\
\hline 6 & A6 & B6 & C6 & D6 & E6 & F6 & G6 \\
\hline 7 & A7 & B7 & $\mathrm{C} 7$ & D7 & E7 & F7 & G7 \\
\hline 8 & A8 & B8 & $\mathrm{C} 8$ & D8 & E8 & F8 & G8 \\
\hline 9 & A9 & B9 & C9 & D9 & E9 & F9 & G9 \\
\hline 10 & A10 & B10 & $\mathrm{C} 10$ & D10 & E10 & F10 & $\mathrm{G} 10$ \\
\hline 11 & A11 & B11 & C11 & D11 & E11 & F11 & G11 \\
\hline 12 & A12 & B12 & C12 & D12 & E12 & F12 & G12 \\
\hline 13 & A13 & B13 & C13 & D13 & E13 & F13 & G13 \\
\hline 14 & A14 & B14 & C14 & D14 & E14 & F14 & G14 \\
\hline 15 & A15 & B15 & C15 & D15 & E15 & F15 & G15 \\
\hline 16 & A16 & B16 & C16 & D16 & E16 & F16 & G16 \\
\hline 17 & A17 & B17 & C17 & D17 & E17 & F17 & G17 \\
\hline 18 & A18 & B18 & C18 & D18 & E18 & F18 & G18 \\
\hline 19 & A19 & B19 & C19 & D19 & E19 & F19 & G19 \\
\hline 20 & A20 & B20 & $\mathrm{C} 20$ & D20 & E20 & F20 & G20 \\
\hline 21 & A21 & B21 & $\mathrm{C} 21$ & D21 & E21 & F21 & G21 \\
\hline 22 & A22 & B22 & $\mathrm{C} 22$ & D22 & E22 & F22 & G22 \\
\hline 23 & A23 & B23 & $\mathrm{C} 23$ & D23 & E23 & F23 & G23 \\
\hline 24 & A24 & B24 & C24 & D24 & E24 & F24 & G24 \\
\hline 25 & A25 & B25 & $\mathrm{C} 25$ & D25 & E25 & F25 & G25 \\
\hline 26 & A26 & B26 & C26 & D26 & E26 & F26 & G26 \\
\hline 27 & A27 & B27 & C27 & D27 & E27 & F27 & G27 \\
\hline 28 & A28 & B28 & C28 & D28 & E28 & F28 & G28 \\
\hline 29 & A29 & B29 & C29 & D29 & E29 & F29 & G29 \\
\hline 30 & A30 & B30 & C30 & D30 & E30 & F30 & G30 \\
\hline 31 & A31 & B31 & C31 & D31 & E31 & F31 & G31 \\
\hline 32 & A32 & B32 & C32 & D32 & E32 & F32 & G32 \\
\hline 33 & A33 & B33 & C33 & D33 & E33 & F33 & G33 \\
\hline 34 & A34 & B34 & C34 & D34 & E34 & F34 & G34 \\
\hline 35 & A35 & B35 & C35 & D35 & E35 & F35 & G35 \\
\hline 36 & A36 & B36 & C36 & D36 & E36 & F36 & G36 \\
\hline 37 & A37 & B37 & C37 & D37 & E37 & F37 & G37 \\
\hline
\end{tabular}

1. A1 is worth 1 if the ship from group $A$ is placed in critical point 1 worth 0 if the ship from group $A$ is not placed in the critical point 1

2. A2 value 1 if the ship from group $A$ is placed in point 2 of the value of 0 if the ship from group $A$ is not placed in the point

3. A3 is worth 1 if the ship from group $A$ is placed in point 3 of the value of 0 if the vessel from group $A$ is not placed in the point

so on for each group of ships up to the decision variable G37
4. G37 is worth 1 if the ship from group $G$ is placed at point 37 of value 0 if the vessel of group $\mathrm{G}$ is not placed in the point of 37

\subsection{Objective function}

The purpose of the function is to minimize the total costs required for operations for a year, by minimizing the number of vessels used in operations. along with the costs, but can still reach all vulnerable points

The operating costs required for each group of vessels are different. In addition, each ship also has a different endurance. With information that in one 
year there are 3 months rest period, then in a year there is a total operating time of 275 days. From the number of days in a year it can be known how many times each vessel operates in a year. Furthermore, it can be seen the costs required for each vessel to operate in a year by multiplying the number of operations carried out by each ship with the cost of each vessel in a single operation, then the formulation of the objective function is as follows:

$$
\begin{aligned}
\text { Minimize }= & 27397521000 \sum_{i=1}^{37} \mathrm{Ai}+41497982500 \sum_{i=1}^{37} \mathrm{Bi}+28715481600 \sum_{i=1}^{37} \mathrm{Ci} \\
& +35764632000 \sum_{i=1}^{37} \mathrm{Di}+30405760800 \sum_{i=1}^{37} \mathrm{Ei}+13657308000 \sum_{i=1}^{37} \mathrm{Fi}
\end{aligned}
$$

\subsection{Constraint determination $\sum_{i=1}^{+28900180800} \mathrm{Gi}$}

There are 4 restrictions used in this study:

a. Guarding Limits for Each Prone Point. Each vulnerable point will be reached by at least one ship.

1. $\mathrm{G} 1+\mathrm{G} 2+\mathrm{F} 1+\mathrm{F} 2+\mathrm{F} 3+\mathrm{F} 4+\mathrm{E} 1+\mathrm{E} 2+\mathrm{D} 1+$ $\mathrm{D} 2+\mathrm{C} 1+\mathrm{C} 2+\mathrm{B} 1+\mathrm{B} 2+\mathrm{B} 3+\mathrm{B} 4+\mathrm{A} 1+\mathrm{A} 2+\mathrm{A} 3+\mathrm{A} 4$ $>=1$;

2. $\mathrm{G} 1+\mathrm{G} 2+\mathrm{G} 3+\mathrm{F} 1+\mathrm{F} 2+\mathrm{F} 3+\mathrm{F} 4+\mathrm{E} 1+\mathrm{E} 2+$ $\mathrm{E} 3+\mathrm{D} 1+\mathrm{D} 2+\mathrm{D} 3+\mathrm{C} 1+\mathrm{C} 2+\mathrm{C} 3+\mathrm{B} 1+\mathrm{B} 2+\mathrm{B} 3+\mathrm{B} 4+$ $A 1+A 2+A 3+A 4>=1$;

3. $\mathrm{G} 2+\mathrm{G} 3+\mathrm{G} 4+\mathrm{F} 1+\mathrm{F} 2+\mathrm{F} 3+\mathrm{F} 4+\mathrm{F} 5+\mathrm{E} 2+$ $\mathrm{E} 3+\mathrm{E} 4+\mathrm{D} 2+\mathrm{D} 3+\mathrm{D} 4+\mathrm{C} 2+\mathrm{C} 3+\mathrm{C} 4+\mathrm{B} 1+\mathrm{B} 2+\mathrm{B} 3$ $+\mathrm{B} 4+\mathrm{A} 1+\mathrm{A} 2+\mathrm{A} 3+\mathrm{A} 4>=1$;

4. $\mathrm{G} 3+\mathrm{G} 4+\mathrm{F} 1+\mathrm{F} 2+\mathrm{F} 3+\mathrm{F} 4+\mathrm{F} 5+\mathrm{F} 6+\mathrm{F} 7+$ $\mathrm{E} 3+\mathrm{E} 4+\mathrm{D} 3+\mathrm{D} 4+\mathrm{C} 3+\mathrm{C} 4+\mathrm{B} 1+\mathrm{B} 2+\mathrm{B} 3+\mathrm{B} 4+\mathrm{B} 5$ $+\mathrm{B} 6+\mathrm{A} 1+\mathrm{A} 2+\mathrm{A} 3+\mathrm{A} 4+\mathrm{A} 5+\mathrm{A} 6>=1 ;$

5. $\mathrm{G} 5+\mathrm{G} 6+\mathrm{G} 7+\mathrm{F} 3+\mathrm{F} 4+\mathrm{F} 5+\mathrm{F} 6+\mathrm{F} 7+\mathrm{F} 8+$ $\mathrm{E} 5+\mathrm{E} 6+\mathrm{E} 7+\mathrm{D} 5+\mathrm{D} 6+\mathrm{D} 7+\mathrm{C} 5+\mathrm{C} 6+\mathrm{C} 7+\mathrm{B} 4+\mathrm{B} 5$ $+\mathrm{B} 6+\mathrm{B} 7+\mathrm{B} 8+\mathrm{A} 4+\mathrm{A} 5+\mathrm{A} 6+\mathrm{A} 7+\mathrm{A} 8>=1$;

6. $\mathrm{G} 5+\mathrm{G} 6+\mathrm{G} 7+\mathrm{F} 4+\mathrm{F} 5+\mathrm{F} 6+\mathrm{F} 7+\mathrm{F} 8+\mathrm{E} 5+$ $\mathrm{E} 6+\mathrm{E} 7+\mathrm{D} 5+\mathrm{D} 6+\mathrm{D} 7+\mathrm{C} 5+\mathrm{C} 6+\mathrm{C} 7+\mathrm{B} 4+\mathrm{B} 5+\mathrm{B} 6$ $+\mathrm{B} 7+\mathrm{A} 4+\mathrm{A} 5+\mathrm{A} 6+\mathrm{A} 7>=1$;

7. $\mathrm{G} 5+\mathrm{G} 6+\mathrm{G} 7+\mathrm{G} 8+\mathrm{F} 4+\mathrm{F} 5+\mathrm{F} 6+\mathrm{F} 7+\mathrm{F} 8+$ $\mathrm{F} 9+\mathrm{E} 5+\mathrm{E} 6+\mathrm{E} 7+\mathrm{E} 8+\mathrm{D} 5+\mathrm{D} 6+\mathrm{D} 7+\mathrm{D} 8+\mathrm{C} 5+\mathrm{C} 6$ $+\mathrm{C} 7+\mathrm{C} 8+\mathrm{B} 5+\mathrm{B} 6+\mathrm{B} 7+\mathrm{B} 8+\mathrm{B} 9+\mathrm{A} 5+\mathrm{A} 6+\mathrm{A} 7+$ $A 8+A 9>=1$;

8. $\mathrm{G} 7+\mathrm{G} 8+\mathrm{F} 5+\mathrm{F} 6+\mathrm{F} 7+\mathrm{F} 8+\mathrm{F} 9+\mathrm{F} 10+\mathrm{F} 11$ $+\mathrm{E} 7+\mathrm{E} 8+\mathrm{D} 7+\mathrm{D} 8+\mathrm{C} 7+\mathrm{C} 8+\mathrm{B} 5+\mathrm{B} 7+\mathrm{B} 8+\mathrm{B} 9+$ $\mathrm{B} 10+\mathrm{A} 5+\mathrm{A} 7+\mathrm{A} 8+9+\mathrm{A} 10>=1 ;$

9. $\mathrm{G} 9+\mathrm{G} 10+\mathrm{F} 7+\mathrm{F} 8+\mathrm{F} 9+\mathrm{F} 10+\mathrm{F} 11+\mathrm{F} 12+$ $\mathrm{E} 9+\mathrm{E} 10+\mathrm{D} 9+\mathrm{D} 10+\mathrm{C} 9+\mathrm{C} 10+\mathrm{B} 7+\mathrm{B} 8+\mathrm{B} 9+\mathrm{B} 10$ $+\mathrm{B} 11+\mathrm{A} 7+\mathrm{A} 8+\mathrm{A} 9+\mathrm{A} 10+\mathrm{A} 11>=1$;
10. $\mathrm{G} 9+\mathrm{G} 10+\mathrm{G} 11+\mathrm{F} 8+\mathrm{F} 9+\mathrm{F} 10+\mathrm{F} 11+\mathrm{F} 12+$ $\mathrm{F} 13+\mathrm{F} 27+\mathrm{E} 9+\mathrm{E} 10+\mathrm{E} 11+\mathrm{D} 9+\mathrm{D} 10+\mathrm{D} 11+\mathrm{C} 9+$ $\mathrm{C} 10+\mathrm{C} 11+\mathrm{B} 8+\mathrm{B} 9+\mathrm{B} 10+\mathrm{B} 11+\mathrm{B} 12+\mathrm{B} 13+\mathrm{B} 27+\mathrm{A} 8$ $+A 9+A 10+A 11+A 12+A 13+A 27>=1 ;$

11. $\mathrm{G} 10+\mathrm{G} 11+\mathrm{G} 12+\mathrm{F} 8+\mathrm{F} 9+\mathrm{F} 10+\mathrm{F} 11+\mathrm{F} 12$ $+\mathrm{F} 13+\mathrm{F} 27+\mathrm{F} 28+\mathrm{E} 10+\mathrm{E} 11+\mathrm{E} 12+\mathrm{D} 10+\mathrm{D} 11+$ $\mathrm{D} 12+\mathrm{C} 10+\mathrm{C} 11+\mathrm{C} 12+\mathrm{B} 9+\mathrm{B} 10+\mathrm{B} 11+\mathrm{B} 12+\mathrm{B} 13$ $+\mathrm{B} 27+\mathrm{A} 9+\mathrm{A} 10+\mathrm{A} 11+\mathrm{A} 12+\mathrm{A} 13+\mathrm{A} 27>=1$;

12. $\mathrm{G} 11+\mathrm{G} 12+\mathrm{G} 13+\mathrm{G} 27+\mathrm{F} 9+\mathrm{F} 10+\mathrm{F} 11+$ $\mathrm{F} 12+\mathrm{F} 13+\mathrm{F} 14+\mathrm{F} 25+\mathrm{F} 26+\mathrm{F} 27+\mathrm{F} 28+\mathrm{E} 11+\mathrm{E} 12$ $+\mathrm{E} 13+\mathrm{E} 27+\mathrm{D} 11+\mathrm{D} 12+\mathrm{D} 13+\mathrm{D} 27+\mathrm{C} 11+\mathrm{C} 12+$ $\mathrm{C} 13+\mathrm{C} 27+\mathrm{B} 10+\mathrm{B} 11+\mathrm{B} 12+\mathrm{B} 13+\mathrm{B} 25+\mathrm{B} 26+$ $\mathrm{B} 27+\mathrm{B} 28+\mathrm{A} 10+\mathrm{A} 11+\mathrm{A} 12+\mathrm{A} 13+\mathrm{A} 25+\mathrm{A} 26+$ $A 27+A 28>=1$;

13. $\mathrm{G} 12+\mathrm{G} 13+\mathrm{F} 10+\mathrm{F} 11+\mathrm{F} 12+\mathrm{F} 13+\mathrm{F} 14+$ $\mathrm{F} 26+\mathrm{E} 12+\mathrm{E} 13+\mathrm{D} 12+\mathrm{D} 13+\mathrm{C} 12+\mathrm{C} 13+\mathrm{B} 10+$ $\mathrm{B} 11+\mathrm{B} 12+\mathrm{B} 13+\mathrm{B} 14+\mathrm{A} 10+\mathrm{A} 11+\mathrm{A} 12+\mathrm{A} 13+\mathrm{A} 14$ $>=1$;

14. $\mathrm{G} 14+\mathrm{F} 12+\mathrm{F} 13+\mathrm{F} 14+\mathrm{F} 15+\mathrm{E} 14+\mathrm{D} 14+$ $\mathrm{C} 14+\mathrm{B} 13+\mathrm{B} 14+\mathrm{B} 15+\mathrm{A} 13+\mathrm{A} 14+\mathrm{A} 15>=1 ;$

15. $\mathrm{G} 15+\mathrm{F} 14+\mathrm{F} 15+\mathrm{F} 16+\mathrm{F} 17+\mathrm{E} 15+\mathrm{D} 15+$ $\mathrm{C} 15+\mathrm{B} 14+\mathrm{B} 15+\mathrm{B} 16+\mathrm{A} 14+\mathrm{A} 15+\mathrm{A} 16>=1$;

16. $\mathrm{G} 16+\mathrm{G} 17+\mathrm{F} 15+\mathrm{F} 16+\mathrm{F} 17+\mathrm{F} 18+\mathrm{E} 16+$ $\mathrm{E} 17+\mathrm{D} 16+\mathrm{D} 17+\mathrm{C} 16+\mathrm{C} 17+\mathrm{B} 15+\mathrm{B} 16+\mathrm{B} 17+$ $\mathrm{A} 15+\mathrm{A} 16+\mathrm{A} 17>=1$;

17. $\mathrm{G} 16+\mathrm{G} 17+\mathrm{F} 15+\mathrm{F} 16+\mathrm{F} 17+\mathrm{F} 18+\mathrm{F} 19+$ $\mathrm{F} 21+\mathrm{F} 22+\mathrm{E} 16+\mathrm{E} 17+\mathrm{D} 16+\mathrm{D} 17+\mathrm{C} 16+\mathrm{C} 17+$ $\mathrm{B} 16+\mathrm{B} 17+\mathrm{B} 18+\mathrm{B} 19+\mathrm{A} 16+\mathrm{A} 17+\mathrm{A} 18+\mathrm{A} 19>=1$; 18. $\mathrm{G} 18+\mathrm{G} 19+\mathrm{F} 16+\mathrm{F} 17+\mathrm{F} 18+\mathrm{F} 19+\mathrm{E} 18+$ $\mathrm{E} 19+\mathrm{D} 18+\mathrm{D} 19+\mathrm{C} 18+\mathrm{C} 19+\mathrm{B} 17+\mathrm{B} 18+\mathrm{B} 19+$ $\mathrm{A} 17+\mathrm{A} 18+\mathrm{A} 19>=1$;

19. $\mathrm{G} 18+\mathrm{G} 19+\mathrm{F} 17+\mathrm{F} 18+\mathrm{F} 19+\mathrm{E} 18+\mathrm{E} 19$ $+\mathrm{D} 18+\mathrm{D} 19+\mathrm{C} 18+\mathrm{C} 19+\mathrm{B} 17+\mathrm{B} 18+\mathrm{B} 19+\mathrm{A} 17+\mathrm{A} 18$ $+\mathrm{A} 19>=1$;

20. $\mathrm{G} 20+\mathrm{F} 20+\mathrm{F} 21+\mathrm{F} 22+\mathrm{E} 20+\mathrm{D} 20+\mathrm{C} 20$ $+\mathrm{B} 20+\mathrm{B} 21+\mathrm{A} 20+\mathrm{A} 21>=1$; 
21. $\mathrm{G} 21+\mathrm{G} 22+\mathrm{F} 17+\mathrm{F} 20+\mathrm{F} 21+\mathrm{F} 22+\mathrm{F} 23+$ $\mathrm{F} 24+\mathrm{E} 21+\mathrm{E} 22+\mathrm{D} 21+\mathrm{D} 22+\mathrm{C} 21+\mathrm{C} 22+\mathrm{B} 20+$ $\mathrm{B} 21+\mathrm{B} 22+\mathrm{B} 23+\mathrm{A} 20+\mathrm{A} 21+\mathrm{A} 22+\mathrm{A} 23>=1 ;$

22. $\mathrm{G} 21+\mathrm{G} 22+\mathrm{F} 17+\mathrm{F} 20+\mathrm{F} 21+\mathrm{F} 22+\mathrm{F} 23+$ $\mathrm{F} 24+\mathrm{E} 21+\mathrm{E} 22+\mathrm{D} 21+\mathrm{D} 22+\mathrm{C} 21+\mathrm{C} 22+\mathrm{B} 21+$ $\mathrm{B} 22+\mathrm{B} 23+\mathrm{B} 24+\mathrm{A} 21+\mathrm{A} 22+\mathrm{A} 23+\mathrm{A} 24>=1$;

23. $\mathrm{G} 23+\mathrm{G} 24+\mathrm{F} 21+\mathrm{F} 22+\mathrm{F} 23+\mathrm{F} 24+\mathrm{E} 23+$ $\mathrm{E} 24+\mathrm{D} 23+\mathrm{D} 24+\mathrm{C} 23+\mathrm{C} 24+\mathrm{B} 21+\mathrm{B} 22+\mathrm{B} 23+\mathrm{B} 24+$ $\mathrm{A} 21+\mathrm{A} 22+\mathrm{A} 23+\mathrm{A} 24>=1$

24. $\mathrm{G} 23+\mathrm{G} 24+\mathrm{F} 21+\mathrm{F} 22+\mathrm{F} 23+\mathrm{F} 24+\mathrm{F} 25+$ $\mathrm{E} 23+\mathrm{E} 24+\mathrm{D} 23+\mathrm{D} 24+\mathrm{C} 23+\mathrm{C} 24+\mathrm{B} 22+\mathrm{B} 23+$ $\mathrm{B} 24+\mathrm{A} 22+\mathrm{A} 23+\mathrm{A} 24>=1$;

25. $\mathrm{G} 25+\mathrm{G} 26+\mathrm{F} 12+\mathrm{F} 24+\mathrm{F} 25+\mathrm{F} 26+\mathrm{F} 27+$ $\mathrm{F} 28+\mathrm{E} 25+\mathrm{E} 26+\mathrm{D} 25+\mathrm{D} 26+\mathrm{C} 25+\mathrm{C} 26+\mathrm{B} 12+$ $\mathrm{B} 25+\mathrm{B} 26+\mathrm{B} 27+\mathrm{B} 28+\mathrm{A} 12+\mathrm{A} 25+\mathrm{A} 26+\mathrm{A} 27+\mathrm{A} 28$ $>=1$;

26. $\mathrm{G} 25+\mathrm{G} 26+\mathrm{G} 28+\mathrm{F} 12+\mathrm{F} 13+\mathrm{F} 25+\mathrm{F} 26+$ $\mathrm{F} 27+\mathrm{F} 28+\mathrm{F} 29+\mathrm{E} 25+\mathrm{E} 26+\mathrm{E} 28+\mathrm{D} 25+\mathrm{D} 26+\mathrm{D} 28$ $+\mathrm{C} 25+\mathrm{C} 26+\mathrm{C} 28+\mathrm{B} 12+\mathrm{B} 25+\mathrm{B} 26+\mathrm{B} 27+\mathrm{B} 28+$ $\mathrm{A} 12+\mathrm{A} 25+\mathrm{A} 26+\mathrm{A} 27+\mathrm{A} 28>=1 ;$

27. $\mathrm{G} 12+\mathrm{G} 27+\mathrm{G} 28+\mathrm{F} 10+\mathrm{F} 11+\mathrm{F} 12+\mathrm{F} 25+$ $\mathrm{F} 26+\mathrm{F} 27+\mathrm{F} 28+\mathrm{F} 29+\mathrm{E} 12+\mathrm{E} 27+\mathrm{E} 28+\mathrm{D} 12+\mathrm{D} 27$ $+\mathrm{D} 28+\mathrm{C} 12+\mathrm{C} 27+\mathrm{C} 28+\mathrm{B} 10+\mathrm{B} 11+\mathrm{B} 12+\mathrm{B} 25+$ $\mathrm{B} 26+\mathrm{B} 27+\mathrm{B} 28+\mathrm{A} 10+\mathrm{A} 11+\mathrm{A} 12+\mathrm{A} 25+\mathrm{A} 26+\mathrm{A} 27$ $+\mathrm{A} 28>=1$;

28. $\mathrm{G} 26+\mathrm{G} 27+\mathrm{G} 28+\mathrm{F} 11+\mathrm{F} 12+\mathrm{F} 25+\mathrm{F} 26+$ $\mathrm{F} 27+\mathrm{F} 28+\mathrm{F} 29+\mathrm{F} 30+\mathrm{E} 26+\mathrm{E} 27+\mathrm{E} 28+\mathrm{D} 26+\mathrm{D} 27$ $+\mathrm{D} 28+\mathrm{C} 26+\mathrm{C} 27+\mathrm{C} 28+\mathrm{B} 12+\mathrm{B} 25+\mathrm{B} 26+\mathrm{B} 27+$ $\mathrm{B} 28+\mathrm{B} 29+\mathrm{B} 30+\mathrm{A} 12+\mathrm{A} 25+\mathrm{A} 26+\mathrm{A} 27+\mathrm{A} 28+\mathrm{A} 29$ $+\mathrm{A} 30>=1$;

29. $\mathrm{G} 29+\mathrm{G} 30+\mathrm{F} 26+\mathrm{F} 27+\mathrm{F} 28+\mathrm{F} 29+\mathrm{F} 30+$ $\mathrm{E} 29+\mathrm{E} 30+\mathrm{D} 29+\mathrm{D} 30+\mathrm{C} 29+\mathrm{C} 30+\mathrm{B} 28+\mathrm{B} 29+$ $\mathrm{B} 30+\mathrm{A} 28+\mathrm{A} 29+\mathrm{A} 30>=1 ;$

30. $\mathrm{G} 29+\mathrm{G} 30+\mathrm{F} 28+\mathrm{F} 29+\mathrm{F} 30+\mathrm{F} 31+\mathrm{E} 29+$ $\mathrm{E} 30+\mathrm{D} 29+\mathrm{D} 30+\mathrm{C} 29+\mathrm{C} 30+\mathrm{B} 28+\mathrm{B} 29+\mathrm{B} 30+$ $\mathrm{A} 28+\mathrm{A} 29+\mathrm{A} 30>=1$;

31. $\mathrm{G} 31+\mathrm{G} 32+\mathrm{F} 30+\mathrm{F} 31+\mathrm{F} 32+\mathrm{F} 33+\mathrm{E} 31+$ $\mathrm{E} 32+\mathrm{D} 31+\mathrm{D} 32+\mathrm{C} 31+\mathrm{C} 32+\mathrm{B} 31+\mathrm{B} 32+\mathrm{A} 31+$ A32 > $=1$;

32. $\mathrm{G} 31+\mathrm{G} 32+\mathrm{F} 31+\mathrm{F} 32+\mathrm{F} 33+\mathrm{E} 31+\mathrm{E} 32+$ $\mathrm{D} 31+\mathrm{D} 32+\mathrm{C} 31+\mathrm{C} 32+\mathrm{B} 31+\mathrm{B} 32+\mathrm{A} 31+\mathrm{A} 32>=1$

33. $\mathrm{G} 33+\mathrm{F} 31+\mathrm{F} 32+\mathrm{F} 33+\mathrm{F} 34+\mathrm{F} 35+\mathrm{E} 33+$ $\mathrm{D} 33+\mathrm{C} 33+\mathrm{B} 33+\mathrm{B} 34+\mathrm{A} 33+\mathrm{A} 34>=1$;

34. $\mathrm{G} 34+\mathrm{G} 35+\mathrm{F} 33+\mathrm{F} 34+\mathrm{F} 35+\mathrm{F} 36+\mathrm{E} 34+$ $\mathrm{E} 35+\mathrm{D} 34+\mathrm{D} 35+\mathrm{C} 34+\mathrm{C} 35+\mathrm{B} 33+\mathrm{B} 34+\mathrm{B} 35+$ $\mathrm{A} 33+\mathrm{A} 34+\mathrm{A} 35>=1$;

35. $\mathrm{G} 34+\mathrm{G} 35+\mathrm{F} 33+\mathrm{F} 34+\mathrm{F} 35+\mathrm{F} 36+\mathrm{F} 37+$ $\mathrm{E} 34+\mathrm{E} 35+\mathrm{D} 34+\mathrm{D} 35+\mathrm{C} 34+\mathrm{C} 35+\mathrm{B} 34+\mathrm{B} 35+$ $\mathrm{B} 36+\mathrm{A} 34+\mathrm{A} 35+\mathrm{A} 36>=1$;
36. $\mathrm{G} 36+\mathrm{G} 37+\mathrm{F} 34+\mathrm{F} 35+\mathrm{F} 36+\mathrm{F} 37+\mathrm{E} 36+$ $\mathrm{E} 37+\mathrm{D} 36+\mathrm{D} 37+\mathrm{C} 36+\mathrm{C} 37+\mathrm{B} 35+\mathrm{B} 36+\mathrm{B} 37+$ $\mathrm{A} 35+\mathrm{A} 36+\mathrm{A} 37>=1$;

37. $\mathrm{G} 36+\mathrm{G} 37+\mathrm{F} 35+\mathrm{F} 36+\mathrm{F} 37+\mathrm{E} 36+\mathrm{E} 37+$ $\mathrm{D} 36+\mathrm{D} 37+\mathrm{C} 36+\mathrm{C} 37+\mathrm{B} 36+\mathrm{B} 37+\mathrm{A} 36+\mathrm{A} 37>=1$

b. Limitation of Ship Placement at Prone Points Each ship from the existing group of vessels can only occupy one vulnerable point, to prevent one vulnerable point from being occupied by more than one ship.

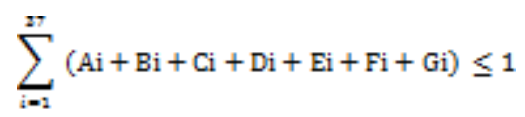

$\mathrm{A} 1+\mathrm{B} 1+\mathrm{C} 1+\mathrm{D} 1+\mathrm{F} 1+\mathrm{G} 1 \leq 1$

$\mathrm{A} 2+\mathrm{B} 2+\mathrm{C} 2+\mathrm{D} 2+\mathrm{F} 2+\mathrm{G} 2 \leq 1$

$\mathrm{A} 3+\mathrm{B} 3+\mathrm{C} 3+\mathrm{D} 3+\mathrm{F} 3+\mathrm{G} 3 \leq 1$

$\mathrm{A} 37+\mathrm{B} 37+\mathrm{C} 37+\mathrm{D} 37+\mathrm{F} 37+\mathrm{G} 37 \leq 1$

c. Limitation of the number of vessels per vessel group

$$
\begin{aligned}
& A 1+A 2+A 3+\ldots+A 37 \leq 1 \\
& B 1+B 2+B 3+\ldots+B 37 \leq 2 \\
& C 1+C 2+C 3+\ldots+C 37 \leq 8 \\
& D 1+D 2+D 3+\ldots+D 37 \leq 3 \\
& E 1+E 2+E 3+\ldots+E 37 \leq 4 \\
& F 1+F 2+F 3+\ldots+F 37 \leq 1 \\
& G 1+G 2+G 3+\ldots+G 37 \leq 1
\end{aligned}
$$

\section{d. Limitation of Binary Numbers}

If the selected ship is worth 1 while if it is not then it is worth $0 . A_{\mathrm{i}}, B_{\mathrm{i}}, C_{\mathrm{i}}, D_{\mathrm{i}}, E_{\mathrm{i}}, F_{\mathrm{i}}, G_{\mathrm{i}} \in\{0,1\} \quad \forall i$

\subsection{Optimizing process}

The process of optimizing the assignment of Indonesian Navy ships in the territorial waters of Riau Islands was assisted by the use of Lingo 11 software. After the model was entered into Lingo 11 , the optimization process was carried out using the solve command. The Value column shows whether the decision variable in question is selected or not in the model. The total number of 
ships used is 12 ships ; $A 17, B 14, B 28, D 4, D 7$, D36, E1, E31, E33, E34, F21, dan G10.

\subsection{Optimization result}

From the optimization results, it was found that the ships assigned were 12 ships. Of the total 20 ships, 12 ships were able to cover all the critical points, so there were savings on the costs of the operations carried out.

Table 3. Result Optimization of Assignment of Indonesian Navy ships

\begin{tabular}{|c|c|c|c|}
\hline KRI Group & Amount Use & Operating cost 1 year (Rp) & Operating cost total (Rp) \\
\hline A & 1 & 27.397 .521 .000 & 27.397 .521 .000 \\
\hline B & 2 & 41.479 .982 .500 & $82 . .959 .965 .000$ \\
\hline C & & 28.715 .481 .600 & 0 \\
\hline D & 3 & 35.764 .632 .000 & 107.293 .896 .000 \\
\hline E & 4 & 30.405 .760 .800 & 121.623 .043 .200 \\
\hline F & 1 & 13.657 .308 .000 & 13.657 .308 .000 \\
\hline G & 1 & 28.900 .180 .800 & 28.900 .180 .800 \\
\hline
\end{tabular}

This cost is more economical than assigning all ships, namely as many as 20 ships which cost Rp. $611,555,766,800$ in one year of operation.

This optimization model has drawbacks, namely the protected hotspots are derived from historical data and from intelligence data. There is a possibility of criminal acts occurring at the vulnerable points that are not recorded on this model. Therefore if there is a new critical point that is not present in this model, then it is necessary to form a new model again by entering the new critical point.

\subsection{Model verification}

Model verification is carried out by manually plotting patrol boat assignments at the hotspots.
The total number of ships used is 12 ships $A 17$, B14, B28, D4, D7, D36, E1, E31, E33, E34, F21, and G10. So the cost needed is Rp. $381,831,914,000$, - with details listed in the following table:

Assigned ships will reach vulnerable points based on known radar range. This model verification is done by entering the optimization results, namely A17, B14, B28, D4, D7, D36, E1, E31, E33, E34, $F 21$, and $G 10$ at the existing critical points. Furthermore, each cell that is affordable by the ship in question will be blocked with certain colors that represent the group of ships. The group A ship is blue. Group B is green. Group D ships are red. Group $E$ is yellow. The group of ships $F$ is purple. The group of $G$ ships is orange. This is done continuously until all ships have been plotted at the assigned point of vulnerability. The results of the verification can be seen in the following table 4 
Table 4. The results of the model verification

\begin{tabular}{|c|c|c|c|c|c|c|c|c|c|c|c|c|c|c|c|c|c|c|c|c|c|c|c|c|c|c|c|c|c|c|c|c|c|c|}
\hline \multirow[t]{2}{*}{ KRI } & \multirow{2}{*}{ Point } & \multicolumn{33}{|c|}{ AFFORDABLE VULNERABLE POINTS } \\
\hline & & & & 3 & & & $\begin{array}{l}6 \quad 7 \\
\end{array}$ & & & & & & & & 11 & & 10 & & & & 22 & & & & $6 \quad 27$ & & 29 & 30 & & & & & & 637 \\
\hline \multirow[t]{3}{*}{ E } & 1 & & 28 & 49 & 821 & 651 & 7420 & & 292 & & 347] & & & & & & & & & & $600 \mid$ & $590 \mid 4$ & 7755 & 104 & 1141 & $6 \longdiv { 4 8 }$ & 518 & & 655 & 6737 & 7738 & 5528 & & \begin{tabular}{|l|l|}
05 & 729 \\
\end{tabular} \\
\hline & 2 & & 0 & 21 & & 371 & 4617 & 200 & 264 & 294 & 319 & 362 & & & & 05 & & & & J9/ & 574 & 5625 & 5214 & 47 4 & 1338 & 8394 & 464 & 486 & 601 & 6197 & 7197 & 9988 & & 71747 \\
\hline & 3 & & 21 & 0 & & 161 & 2515 & 85 & 243 & 273 & 298 & 341 & & & & 962 & & 110 & 640 & 561 & 551 & 5415 & 2114 & $26 \mid 42$ & 2.236 & 7399 & 469 & & 606 & 624 & 7248 & 3038 & & 50726 \\
\hline \multirow[t]{3}{*}{$D$} & & & & & & 339 & 2 & 15. & 210 & 240 & 265 & 308 & 33 & & & 659 & 659 & 685 & 613 & 528 & 518 & 5084 & 4883 & 933 & \begin{tabular}{l|l}
3933 \\
39
\end{tabular} & 4366 & 436 & & 573 & 5916 & 6917 & 7707 & & 17693 \\
\hline & & & & 116 & & 0 & 93 & 69 & 127 & 157 & 182 & 26 & & & & $3 \mid 51$ & 50 & DOL & 530 & 44 & 435 & 4254 & & 103 & 0625 & 1283 & 353 & 375 & 490 & 5086 & 6086 & 687 & & 34610 \\
\hline & 6 & & 146 & 125 & 92 & 9 & 02 & 11 & 176 & 206 & 231 & $2 / 4$ & 99 & & 45 & 256 & 625 & 651 & $J / 9$ & 49 & 484 & $474 \mid$ & & 593 & 5530 & 0332 & 402 & & 539 & 5576 & 6577 & 73617 & & 83659 \\
\hline \multirow[t]{3}{*}{ D } & 7 & & 173 & 152 & & & 170 & 33 & 91 & ILI & 146 & 189 & 14: & & $9 / 4$ & 7]47, & 240 & 566 & 444 & 409 & 399 & 389 3 & $369 / 2$ & 742 & 70212 & $5[247$ & 317 & 339 & 454 & 472.5 & 5726 & 5516 & & 881564 \\
\hline & 8 & & 4206 & 185 & 152 & 691 & 183 & 0 & 58 & 88 & 113 & 156 & 81 [1 & & 644 & 44 & 507 & 533 & 461 & 376 & 366 & 3563 & 3362 & 412 & 3721. & 5247 & 317 & 339 & 454 & 4725 & 5726 & 5516 & & 65241 \\
\hline & 9 & 22 & 2264 & 243 & & 271 & 769 & 58 & 0 & 30 & 55 & 98 & & & 83 & 638 & 499 & 475 & 403 & 318 & 308 & 2982 & 7781 & 831 & 7912 & 4156 & 226 & & 363 & 381. & 4815 & 6005 & & 12388 \\
\hline \multirow[t]{4}{*}{ G } & 10 & 12 & 2294 & 273 & & 572 & 0612 & 88 & 30 & 0 & 25 & 68 & 3 & 32 & 833 & 635 & 419 & 45 & 373 & 288 & 278 & 2682 & 2481 & 531 & 4994 & 126 & 196 & 218 & 333 & 3514 & 4515 & 5305 & & 82358 \\
\hline & 11 & & & 298 & & 822 & 3114 & 11 & 55 & 25 & 0 & 43 & 8 & & 312 & 133. & 394 & 420 & 348 & 263 & 253 & 2432 & 2231 & 281 & 2469 & 101 & 171 & 193 & 308 & 326 & 4265 & 6055 & & $52[428$ \\
\hline & 12 & & 362 & 341 & & 252 & \begin{tabular}{l|l|l|}
74 & 18 \\
\end{tabular} & 15 & 98 & 68 & 43 & 0 & 5 & & 012 & $8 \mid 28$ & 35. & 377 & 310 & 220 & 210 & 2001 & 180 & 358 & 1126 & 58 & 128 & & 261 & 2793 & $379 \mid$ & 1584 & & $14 \mid 4200$ \\
\hline & 13 & & 5387 & 366 & & 502 & 9921 & 18. & 123 & 93 & 68 & 25 & 0 & & 512 & 326 & 360 & 352 & 315 & 200 & 213 & 2102 & & 451 & $\begin{array}{lll}06 & 13 \\
\end{array}$ & 1163 & 233 & 255 & 370 & 388 & 4885 & 6675 & & $74 \mid 350$ \\
\hline \multirow[t]{3}{*}{$B$} & & & & 446 & & 303 & 7920 & 26. & 203 & 173 & 148 & 105 & & & 51 & 318 & 246 & 272 & 340 & 255 & 255 & 2602 & 5881 & 801 & 4519 & 6228 & 298 & 320 & 435 & 453 & 5536 & 6326 & & 09385 \\
\hline & 15 & 60 & 532 & 511 & 4783 & 954 & 4435 & 32 & 268 & 238 & 213 & 170 & 45 & & 17 & & 18. & 207 & 306 & 233 & 223 & 2502 & 2002 & 0011 & 8517. & 5207 & 277 & 299 & 414 & 4325 & 5326 & 6116 & & 29405 \\
\hline & 16 & 38 & & 589 & & 735 & 2243 & 40. & 346 & 316 & 291 & 248 & 23 & & $8]$ & 40 & 103 & 129 & 225 & 155 & 180 & 185 & 1901 & 6511 & 55151 & 0182 & 252 & 274 & 389 & 4075 & 5075 & 6866 & & 94370 \\
\hline \multirow[t]{4}{*}{ A } & $1 /$ & 78 & & 629 & & 135 & 6247 & & 386 & 356 & 331 & 288 & 63 & & 84 & 0 & 63 & 89 & 235 & 110 & 120 & 1551 & 1651 & 701 & \begin{tabular}{l|l|}
68 & 16 \\
\end{tabular} & 71999 & 269 & 291 & 406 & 424 & 5246 & 6036 & 253 & 19225 \\
\hline & 18 & 41 & 1713 & 692 & & 7766 & 2554 & 50 & 449 & 419 & 394 & 351 & 26 & & & 363 & 0 & 26 & $1 \sqrt{1}$ & 151 & 130 & 1902 & 2032 & 332 & 31 19: & 3225 & 205 & 317 & 432 & 450 & 5506 & 6296 & & 57333 \\
\hline & 19 & tor & 7739 & 718 & & 026 & 5156 & 53. & 475 & 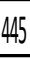 & 420 & 377 & 52 & & & 98 & 20 & 0 & 120 & 142 & 135 & 1992 & 2222 & 122 & 15211 & 9251 & 321 & 343 & 458 & 476 & 576 & 5556 & & 76352 \\
\hline & & & & 646 & & 305 & 7949 & & 403 & TIJ & 348 & 310 & 15 & & 612 & 523 & 151 & 125 & 0 & 85 & 110 & 174 & $197 \mid 2$ & 973 & $\begin{array}{ll}36 & 38 \\
\end{array}$ & 1413 & 483 & 505 & 407 & 425 & 5256 & 6046 & & 51327 \\
\hline \multirow[t]{7}{*}{$F$} & & & & 561 & & 454 & 9440 & & 318 & 288 & 263 & 220 & 30 & & 31 & & & 122 & & $v$ & 25 & 891 & 122 & 122 & 5130 & $6 \quad 291$ & 361 & 322 & & 425 & 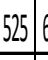 & 60 & & 60242 \\
\hline & 22 & 00 & 5574 & 551 & & 1354 & 8439 & 360 & 308 & 278 & 253 & 210 & 13. & & 31 & 012 & & 135 & 110 & 25 & 0 & 64 & 871 & 872 & 2628 & 1313 & 383 & 405 & 297 & 315 & 4154 & 9945 & & 41217 \\
\hline & 23 & 90 & & 541 & & 2514 & $\begin{array}{ll}7438 \\
\end{array}$ & 35 & 298 & 268 & 243 & 200 & 10 & & 01 & 515 & 190 & 199 & $1 / 4$ & 89 & 64 & 0 & 231 & 231 & 6221 & 7210 & 280 & 302 & 233 & 251 & 351 & 3014 & 2. & 77153 \\
\hline & 24 & 175 & & 521 & & 0054 & 5436 & & 278 & 248 & 223 & 180 & 05 & & & 016 & 20 & 222 & $19 /$ & 112 & 87 & 23 & 01 & 001 & $\begin{array}{ll}39 & 19 . \\
\end{array}$ & 4184 & 254 & 276 & 210 & 228 & $407 \mid$ & 1865 & 608. & $\begin{array}{l}54 \quad 130 \\
\end{array}$ \\
\hline & 25 & 510 & 447 & 426 & & 3103 & $\begin{array}{ll}5927 \\
\end{array}$ & 24. & 183 & 153 & 1288 & 85 & 45. & & & $5 \mid 17$ & {$[3]$} & 212 & LJI & 211 & 187 & 123 & 100 & & 994 & 75 & 145 & 167 & 282 & 300 & $400 \mid$ & 7795 & & 29205 \\
\hline & 26 & 471 & & 422 & & 3063 & 5527 & 23 & 179 & 149 & 124 & 81 & 06 & & & $5 \mid 16$ & {$[\langle 3$.} & 215 & 336 & 251 & 226 & 162 & 139 & 39 & 055 & 40 & 110 & 132 & 247 & 265 & 365 & 444 & 100 & 68244 \\
\hline & 21 & & & & & 513 & 0021 & 211 & 124 & 94 & 69 & 26 & 31 & & & 16 & 19 & 219 & 381 & 300 & 281 & 217 & 194 & 945 & 50 & 32 & 102 & 124 & 239 & 257 & 357 | & 436 & & 23299 \\
\hline \multirow[t]{3}{*}{$B$} & & & & 399 & 3662 & 2833 & 3222 & & 156 & 126 & 101 & & 00 & & 11 & 219 & 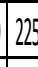 & 251 & 710 & $2 \sqrt{1}$ & 313 & 2101 & 1847 & 754 & 1032 & 0 & 70 & 92 & 207 & 225 & 325 & 4044 & & 04280 \\
\hline & 29 & & & 469 & & 5314 & 0231 & 31 & 226 & 196 & 1711 & 128 & 33 & & & 226 & 159 & 321 & 400 & 361 & 383 & 28012 & 2541 & 451 & 1010 & 270 & 0 & 22 & 137 & 155 & 255 & 3343 & 50 & 74350 \\
\hline & 30 & & & 491 & & 754 & 2433 & 33. & 248 & 218 & 193 & 243 & 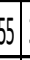 & & 9 & & 31 & 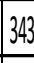 & 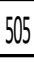 & 322 & 405 & 302 & 7761 & 1671 & 3212 & 492 & 22 & 0 & 115 & 133 & 233 & 3123 & & 96372 \\
\hline \multirow[t]{2}{*}{$\mathrm{E}$} & 31 & 555 & & 6066 & & 1905 & 3945 & & 363 & 333 & 308 & 261 & 70 & & & $9 \mid 40$ & 43 & 458 & 07 & & 297 & 233 & 2102 & 822 & $47 \quad 23$. & 9207 & 137 & 115 & 0 & 18 & 118 & 1972 & & 94270 \\
\hline & 32 & 773 & & 624. & & & 57 47 & & 381 & 351 & 326 & & & & & & 45 & & 25 & 42 & 315 & 251 & 2813 & & 6525 & 71225 & 155 & 133 & 18 & 0 & 100 & 1792 & & 99275 \\
\hline $\mathrm{E}$ & 33 & 73 & & 724 & & 6086 & 5757 & & 481 & 451 & 426 & & 88 & & & 7152 & 55 & 576 & blb & $5 / 5$ & 415 & 351 & 407 / & 1003 & 655 & 7325 & 255 & 233 & 118 & 100 & 0 & 79 & & 70294 \\
\hline$E$ & 34 & 852 & & 803 & & & $\begin{array}{l}3665 \\
\end{array}$ & & 560 & 530 & 505 & & $0 / 1$ & & & & 102 & 655 & 004 & & 494 & 430 & $486 \mid 4$ & 1794 & $\begin{array}{l}44 \\
431\end{array}$ & \begin{tabular}{l|l|l}
6404 \\
\end{tabular} & 334 & 312 & 197 & 179 & 79 & 0 & & $\begin{array}{lll}10 & 134 \\
\end{array}$ \\
\hline & 35 & & & 825 & & 2097 & & & 582 & JuL & 527 & $\begin{array}{l}+00 \\
\end{array}$ & of & & & 06 & 00 & $v i r$ & $v<v$ & & 516 & 452 & 5085 & 014 & 66456 & 8426 & 356 & 334 & 219 & 201 & & 22 & & 8112 \\
\hline$D$ & 36 & & & 750 & & 3346 & & & 412 & Jut & 452 & 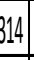 & 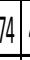 & & & & 定 & 6 & & & 241 & 177 & & 292 & & & 374 & 396 & & & & 110 & & \\
\hline & 37 & 29 & & 7266 & & 1010 & 5956 & & 388 & & 428 & 290 & & & & 7022 & & & & & 217 & 153 & & & 4429 & & 350 & 372 & 270 & & & & & 40 \\
\hline
\end{tabular}

\subsection{Sensitivity analysis}

Sensitivity analysis is done on this model due

to uncertain real conditions. The real uncertain condition that often occurs in marine security operations is that the availability of fuel received by operating vessels is sometimes less than the 
capacity required by the ship. This will result in the ship's endurance also decreasing too. So that the time the ship operates on the sea is shorter than normal conditions. Because the operating time is shorter then the operations needed in a year are more so that the costs incurred will be greater.

Sensitivity analysis is carried out on the objective function coefficient which is the cost needed in a year. Sensitivity analysis in the form of post optimality analysis, namely sensitivity analysis carried out after obtaining the optimal solution. The objective function coefficient, which is operating costs in a year, will increase due to the increasing number of operations caused by the reduced endurance of the ship. Calculations performed will see to what extent the cost changes reach the greatest value due to the reduction in fuel received. From the sensitivity analysis conducted, it is known that the model produces the highest cost output if the fuel received by the ship is $89.14 \%$ of the fuel capacity on the ship. The resulting cost is Rp. $463,621,411,040,-$. Where this cost is greater than the optimal solution without any reduction in fuel received by the ship, which is $\mathrm{Rp}$. $381,831,914,000$ This higher cost is caused by the frequency of operations carried out by ships which are getting bigger in a year.

\section{CONCLUSION}

a. The number of ships needed to cover all vulnerable points is 12 ships

b. The total cost needed in a 1-year operation is Rp. $381,831,914,000$, -. This fee is smaller than using the entire ship, which is 20 ships at a cost of Rp. $611,555,766,800$ a year

c. This model is flexible, where the existing vulnerable points can change. So if there is a new critical point or there is a vulnerable point removed in this model then it is necessary to formulate the model again because the new optimization results obtained will likely be different d. In this study, the problems are discrete. Namely formulating the existing critical points as a priority for safeguards. The more optimal condition is to use a continuous approach because the existing problems are the selection of locations in the continuous area, namely the territorial waters, where the placement location can be placed at all points in the waters

\section{ACKNOWLEDGMENTS}

Thank you, the writer prays to the presence of Allah SWT who has bestowed His Grace and Gift, so that the writing of this Final Project can be completed even though there are challenges and obstacles, the author receives many useful assistance and guidance so that this Final Project can be completed on time, and on this occasion the authors convey many thanks to the supervisors, STTAL commanders and staff, co-operation operations staff, gusamlabar operations staff and all colleagues who helped complete this paper. With all the humbleness and limited knowledge they have, we realize that there are still many shortcomings and far from perfection in the writing of this final assignment, therefore, critics and suggestions from the reader are very authors hope for the improvement of this paper and can provide input for the authors who will come, for improvement. Finally, hopefully the results of writing this Final Project can be useful for the development of the repertoire of knowledge for writers in particular and for readers in general.

\section{REFERENCES}

A,Hamdan, 2003. Operation Research, An Introduction Seventh Edition. Prentice Hall, New Jersey USA.

A,Hamzah, 1984, Marine Territory and Indonesian Waters. Akademia Pressindo, Jakarta.

Asops Kasal, 2005, The concept of the Navy's Operation in the context of Enforcing Sovereignty and Security in the Sea, FPSO, Jakarta. 
Asops Kasal, 2004, Ocean Safety Operation Manual.

Ahmad Lutfi, 2010, Determination of ideal number and optimization of the assignment of elements of ships and aircraft of the Navy maritime patrol in selective prone areas in order to operate the eastern fleet security, STTAL Bachelor Final Project, Surabaya

Daskin, 2008, What You Should Know About Location Modeling
Firmanto, H, 2006,operation research, Solver : Lecture module on Sea Transportation, Faculty of Marine Technology, ITS, Surabaya

Supiyanto, 2009, Optimization Model of Patrol Ship Composition in Eastern fleet Marine Security Operations with Goal Programming Approach, STTAL Bachelor Final Project, Surabaya

Toregas, 1971, Binary Logic Solutions to a Class of Location Problems 